\title{
Performance Tradeoffs by Power Control in Wireless Ad-Hoc Networks
}

\author{
Giorgia V. Rossi and Kin K. Leung \\ Department of Electrical and Electronic Engineering \\ Imperial College, London, United Kingdom \\ Email: \{giorgia.rossi12, kin.leung\}@imperial.ac.uk
}

\begin{abstract}
Topology control for ad-hoc networks is crucial due to the absence of a fixed infrastructure that can guarantee satisfactory connectivity among communication nodes. In this paper, we study the impacts of adjustment of transmission power as a means to control the network topology (connectivity) to the signal-to-interference-plus-noise (SINR) ratio and the number of hops between two nodes, where the SINR and the hop count are highly related to the network throughput and packet delay, respectively. We investigate three distinct topologies: fully, minimally and moderately connected networks. Our results show that adjusting the power level is a very effective way to control the network topology, while enabling to achieve the desirable tradeoffs among SINR and hop count.
\end{abstract}

Keywords-Ad-hoc network; Topology Control; Power Controlt; Signal-to-noise ratio; Connectivity.

\section{INTRODUCTION}

In this paper, we study the topology control problem of Wireless Ad-Hoc networks through adjustments of transmit power. The main goal of this work is to highlight the possible trade-off between transmit power, the signal-to-interferenceplus-noise ratio (SINR) and the number of (transmission) hops from a node to another in the network. It is well known that the hop count and the SINR are strongly related to packet delay and data rate in the network, respectively. To the best of our knowledge, we are not aware of other studies that characterize the performance trade-off between the SINR (data rate) and hop count (delay) by use of power control. Finding the minimum power required for network connectivity can provide benefits for military applications in battlefields, where soldiers are often deployed over hostile and isolated environments.

Wireless ad-hoc networks consist mostly of moving nodes (including vehicles) that need to communicate among themselves without any fixed infrastructure. Due to the lack of infrastructure, every single node has to be able to relay data to other nodes at the same time as it transmits and receives its own data. Consequently, control of the network topology is of vital importance. Power adjustments can be used to create the most suitable network topology using the minimum power level [1], [2], [3], [4]. The authors in [2] propose an algorithm of transmit power adjustment, based on a minimum spanning tree, where the location of every node in the graph (network) is assumed to be known either by use of a GPS or other modes as in [1] and [4]. In [3] an algorithm is presented that does not assume a priori knowledge of the node positions. After assigning a time slot to every node, each node broadcasts a beacon packet at the maximum transmission power level. Based on the average received power levels, a node is then able to find its closest nodes. The only criterion for connectivity between two nodes is based on the received power level and interference is not taken into account. Connectivity for an adhoc network based on signal-to-interference-plus-noise ratio (SINR) is presented in [5]. In order to establish a link, it is required that the SINR at the receiver be above a given threshold. However, the study in [5] does not consider packet delay as a parameter of network quality. The capacity within a network after applying power management is analysed in [6], but does not take into account the delay due to multihop transmissions. In [7], the authors outline an algorithm that takes into account the SINR constraint for connectivity, which is composed of two parts that are invoked alternatively. However, parameters related to delay in the network are not considered. Additionally, the authors in [7] assume that a node is capable of being an interfering node if and only if it is within the range of the receiver. However, even for transmitters outside the range of the receiver, the transmission can be compromised when the other nodes transmit at the same time and consequently the sum of all interferences may cause the SINR at the receiver to fall below the threshold.

We study the effects of transmission power control as a means to control network topology, link quality and hop counts between pairs of nodes in the network. To illustrate the possible performance tradeoffs among these parameters, three distinct topologies are studied. The first one is the fully connected topology (FC) where all nodes transmit at the same maximum power level, so that every node can communicate directly (i.e., one-hop away) to all other nodes. The second topology is referred to as the minimally connected topology (MiC) where each node transmits at a locally minimum power level (which can be different from that of another node) so that all nodes can communicate with at least one other node in the network. Note that in this case some nodes in the network might still be connected via multiple hops. The third topology, referred to as the moderately connected topology $(\mathrm{MoC})$, is closely related to the MiC topology. However, in this case, the transmitting power is set to the same level for every node and equals the maximum value of the $\mathrm{MiC}$ topology. Given these three topologies, we focus on two different scenarios: 1) a network in which the transmitting power is chosen locally for every node, and 2) a network in which the value of transmitting power is the same for all the nodes within the network. In both cases, we study the SINR to find the minimum threshold that is necessary to keep the network connected. The relationships among SINR, connectivity, transmitting power and link quality 
are then studied. Finally, a tool to control the topology based on the network requirements in terms of SINR is proposed.

\section{SYSTEM MODEL}

\section{A. Assumptions}

Before outlining the method and algorithms used in this work, let us highlight our assumptions.

We assume that the network is stacic or it changes slowly [3], such that it can be modelled as quasi-stationary. This means that the nodes in all the three different scenarios we study (FC, MiC and MoC topology) are generally assumed to be pedestrian.

The ad-hoc network under study is modelled by an undirected graph where communication nodes (devices) are nodes in the graph and an edge connecting two nodes represents the connectivity between them. Only undirected graphs are considered, that is, all edge-relations on vertex pair are symmetric. In this work we consider only the connectivity constraint.

The antennas placed on the devices used in our context are supposed to be omni-directional. This means that they radiate the radio wave power uniformly in all directions of the plane. Connectivity between any two nodes is defined as the received signal power at the receiving end and is greater or equal to a given threshold $\mathrm{S}$ such that

$$
P_{i j}^{t}-\gamma_{i j} \geq S
$$

where $P_{i j}^{t}$ is the power transmitted by node $i$ to node $j, y_{i j}$ is the path loss along the path between the two nodes and $\mathrm{S}$ is the sensitivity of the receiver placed at node $j$. The relation above means that the successful reception of a transmitted signal depends on the previously listed parameters, that is the transmission power of node $i$ has to be greater than the sum of the path-loss along the path to reach the other node and the sensitivity of the receiver on node $j$. We note that the requirement in (1) is expressed in decibels $(\mathrm{dB})$.

The sensitivity $S$ is defined as the threshold signal strength needed for reception and is assumed to be an a priori known constant and equal for all nodes.

We do not need to assume that every device has a GPS; hence it is not required to know the exact coordinates of each node in the network to implement the topology control. The new idea is to generalize the algorithm and analyse a scenario in which it is possible to use the path-loss matrix of the entire network. The last assumption needed in this work is to calculate the path-loss matrix of the network through the following steps:

1) The node $\mathrm{i}$ broadcasts a beacon packet at maximum power with an omnidirectional antenna. This process is repeated for every single node of the network, paying attention not to do simultaneously in order not to generate any collision between the beacon packets.

2) Every node $j(j \neq i)$ that receives the signal evaluates the path-loss along the path. The path-loss is defined as the loss in signal strength of the electro-magnetic wave. Node $j$ has to calculate the difference between the maximum signal strength of the beacon packet and the received signal strength. We assume that in every network the maximum transmitting power is set to be the same for every node.

3) Node $j$ sends back a signal to node $i$ with the information about the path-loss they have just calculated.

4) This process is repeated iteratively for every node of the network and assuming that they are all able to exchange the information it is possible to create a path loss matrix for the entire network, and every node is aware of this matrix.

In general, the energy of a signal decreases with distance such that the received power is equal to

$$
P^{r}=\frac{k P^{t}}{d_{i j}{ }^{\alpha}} .
$$

where $P^{r}$ represents the power of a signal that arrives at a receiver and $P^{t}$ the initial power used to transmit the signal. $P^{r}$ decreases to the power of the distance $d_{i j}$ between the transmitting node and the receiving node.

For the simulations, the path-loss is calculated from the Friss transmission equation, i.e.

$$
\frac{P^{r}}{P^{t}}=G_{t} G_{r}\left(\frac{\lambda}{4 \pi R}\right)^{2} .
$$

where $G_{t}$ and $G_{r}$ are, respectively, the gain of the transmit antenna and the gain of the receive antenna. $R$ represents the distance between two antennas and $\lambda$ the wavelength of the signal transmitted. The path loss is proportional to the square of the distance $R$ between the transmitter and receiver, and also proportional to the square of the frequency $f$ of the radio signal. Formally:

$$
\gamma=\left(\frac{4 \pi f R}{c}\right)^{2},
$$

where $c$ is the speed of light. Usually it is more convenient to express the equation in $\mathrm{dB}$.

\section{B. Fully Connected (FC) Network}

To establish a network topology as a baseline for comparison, the first step is to generate a fully connected (FC) topology by using the same transmission power at all nodes. An algorithm for generating the FC topology for a network located in a given geographic area is provided as follows.

1) Identify the size of the square, geographical area that covers all nodes in the network. (We assume it is a square area of $A=500 \mathrm{~m}^{2}$ in our numerical examples.)

2) Assume that two nodes $a$ and $b$ are placed at the opposite corners of $A$, in order to consider the maximum possible distance between two nodes within the network.

3) 3. The minimum transmission power for nodes $a$ and $b$ is calculated so that the received power requirement in (1) is satisfied. Given the maximum distance between nodes $a$ and $b$, this power level is the highest among any two nodes to satisfy (1) for connectivity. Therefore, the power level is denoted by $P_{\max }$. 
4) Set the transmission power to be $P_{\max }$ at all nodes. Since only path loss is considered in this study, such uniform transmission power at all nodes provides a fully connected network.

By definition, in the fully connected topology, each node can reach any other node in one hop.

\section{Minimally Connected $(\mathrm{MiC})$ Network}

To illustrate the effects of topology control by power control, we propose an algorithm to choose the transmission power for each node in order to achieve minimal connectivity among all nodes in a given network. That is, all nodes are connected, perhaps via multi-hops, while the total amount of transmission powers at all nodes is minimised. The Minimally Connected (MiC) network serves as a baseline case for comparison with other topologies. A proof on the optimality of the algorithm is given in [1].

Assuming that the path-loss matrix with elements representing the path loss of any pair of nodes has been obtained by the technique presented in the last subsection, the algorithm for constructing the $\mathrm{MiC}$ network consists of the following steps:

1) Sort all the elements in the upper (or lower) triangular sub-matrix of the path-loss matrix in the ascending order and put them as an ordered list $L$.

2) As an initialization step, the transmission power for each node is fixed to be a fixed value. Treat every node as a cluster and as a result, there are $M$ node clusters for the network with $M$ nodes.

3) Identify the first (smallest) path-loss element in the list $L$ and the corresponding node pairs associated with the path-loss element, say nodes $i$ and $j$.

4) If nodes $i$ and $j$ belong the same cluster, go to Step 6 . Otherwise, they belong to two different clusters. Then, merge the two clusters by increasing the transmission power for nodes $i$ and $j$ from their current values to the minimum power such that (1) can be satisfied.

5) Remove the first element from the list $L$.

6) Repeat from Step 3 if there are more than one node clusters remaining. Otherwise, the single cluster includes all nodes in the connected network.

The network topology generated by the above algorithm provides only one single path from any node to another node in the network, thus being referred to as the minimally connected network. As such, the total transmission power for all nodes is minimised.

\section{Moderately Connected (MoC) Network}

As an alternative, we obtain and study the third network topology with a moderate degree of connectivity among nodes, thus referred to as the Moderately Connected (MoC) network. Specifically, the MoC topology is obtained based on the MiC network generated above, but all nodes transmit at the same power level, which is set to be equal to the maximum power among all nodes in the $\mathrm{MiC}$ topology. Clearly, by using power levels higher than the minimum as in the MiC topology, the degree of connectivity for the MoC network is expected to lie between those of the Fully Connected and MiC networks.

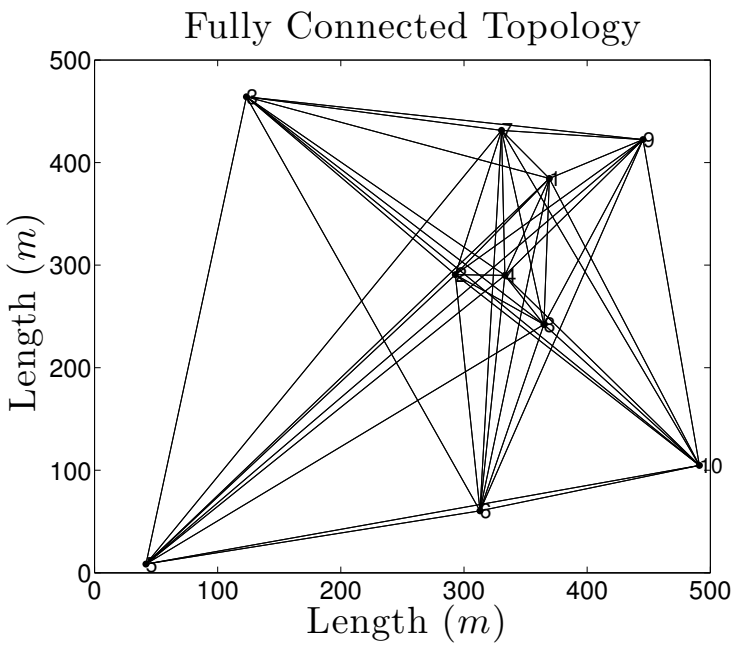

Fig. 1. Example of 10 nodes graph in a FC topology in a square area of $500 \mathrm{~m}^{2}$.

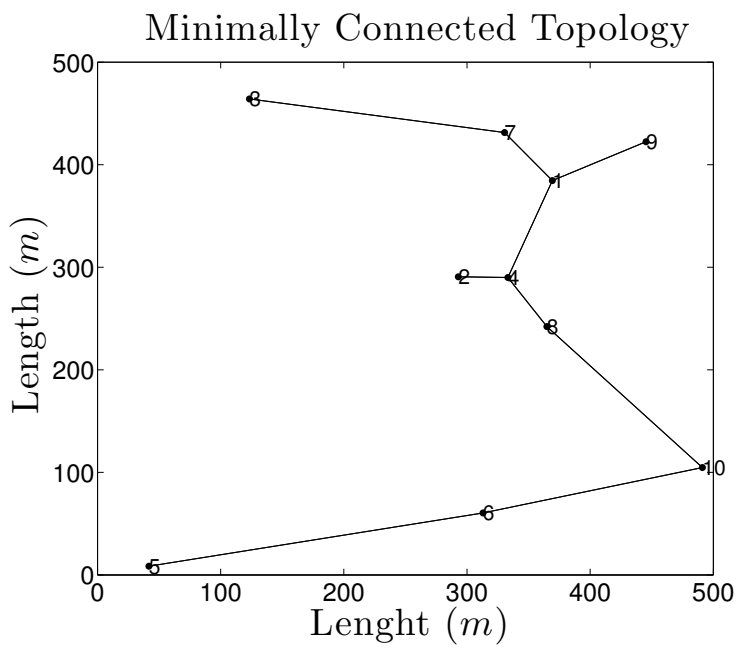

Fig. 2. Example of 10 nodes graph using MiC in a square area of $500 \mathrm{~m}^{2}$.

\section{E. Signal to Interference plus Noise Ratio}

In wireless communication, the SINR is used to measure the quality of wireless connections. This means that a signal can be correctly decoded by the intended receiver only if the ratio between the sensed power of the actual signal to be received and the sum of all power levels experienced due to other signals concurrently transmitted (plus an ambient noise power level) is above a certain hardware-dependent threshold. Usually, this threshold is defined as $\beta$ and consequently:

$$
\frac{P^{r}}{I+N} \geq \beta
$$

with $I$ the interference generated by simultaneous transmissions in the network and $\mathrm{N}$ the noise power. More formally, the SINR at a receiver $\mathrm{j}$ must exceed a given threshold $\beta$ :

$$
S I N R_{j}=\frac{P_{i j}^{r}}{\sum_{m \neq i} P_{m j}^{r}+N} \geq \beta .
$$

$P_{i j}^{r}$ and $P_{m j}$ are, respectively, the received powers of the transmissions between node $i$ and $j$, and between node $i$ and 
$m$.

\section{NUMERICAL STUDY AND DiscUSSION}

We investigate and understand the trade-off among the transmitting power, the SINR and the number of hops between nodes.

Regarding the power metrics (Fig. 3), we can see that the power levels of each node are set to the maximum level $\left(P_{\max }\right)$ in the FC case. The MiC graph, on the other hand, is at the minimum possible level of average transmission power for any connected topology possible. Moreover in MiC case the power level decreases with the increase in node density, because the nodes are increasingly closer ever closer, so they need less power to be connected. If the minimum transmitting power is instead fixed (MoC), consequently the used power level would be slightly higher compared with the previous scenario.

In the FC topology the average hop count is equal to one (Fig. 4). The reason of this is that by using the maximum transmit power all the nodes can reach each other using just a single hop. On the other hand, in the MiC topology, the link metrics are at the maximum possible level for any connected topology possible. This is because it is a minimally connected topology. A significant consequence is the increasing delay in the communications between the nodes. In the MoC topology instead the number of hops is lower than in the MiC topology.

Fig. 5 shows that the quality of the connection increases when power adjustment is used to control the topology (MiC and $\mathrm{MoC}$ topologies). In spite of having higher transmission power in the FC topology, the quality of the connections (in terms of SINR) in this scenario is lower than in MiC and $\mathrm{MoC}$ topologies, due to the fact that high level of interference is generated. Referring to (6), it is possible to observe that high transmission power used to connect all the nodes in the network leads to a great value of interference. Thus the overall quality of the network connectivity is maybe reduced if high transmission power is used depending on the network topology. The SINR could therefore be used to characterise any random network. Depending on the number of nodes in a network area, a particular SINR can either hint at a highly connected topology or a sparsely connected topology.

Depending on the requirements of the network, it is sometimes important to reduce the power levels since battery life is a local resource of each node (i.e. military applications) Note that for VANETs this is not usually applicable. However, there might still be scenarios even for VANETs where power limitation is desirable.

In order to summarise the three performance metrics, namely transmitting power, hops count and throughput, in a single parameter we introduce $\mathrm{U}$ as

$$
U=\frac{\psi}{P^{t}}+\frac{\phi}{T}+\rho \lambda
$$

Where $\psi, \phi$ and $\rho$ are weight factors and $\mathrm{U}$ is affected by the three basic parameters we have studied: average transmitting power $\left(P^{t}\right)$, average hops count $(T)$ and average throughput $(\lambda)$. The greater the power used to transmit or the number of hops needed to connect two nodes, the worse the performance; on the other hand the Utility function is optimised when the

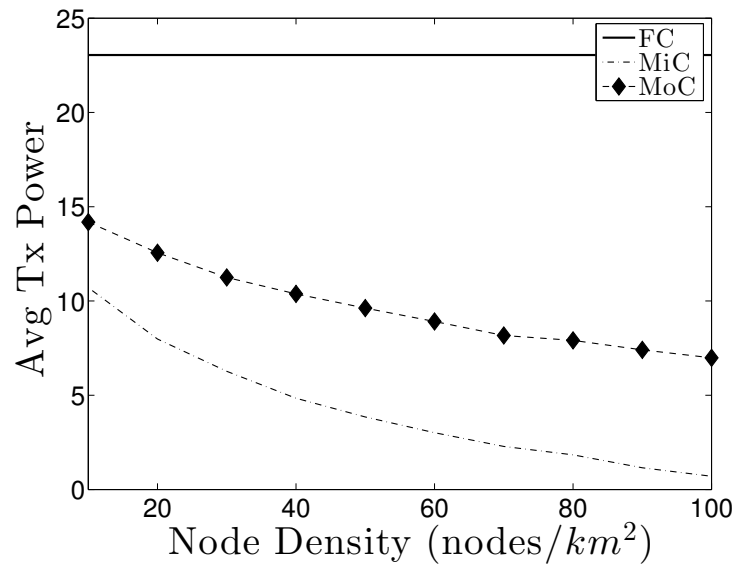

Fig. 3. Average Transmitting Power as a function of the number of nodes.

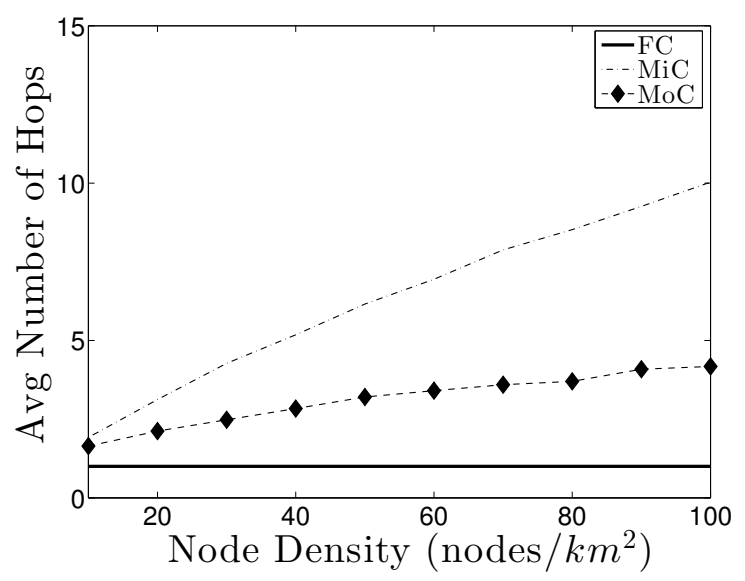

Fig. 4. Average Number of Hops as a function of the number of nodes.

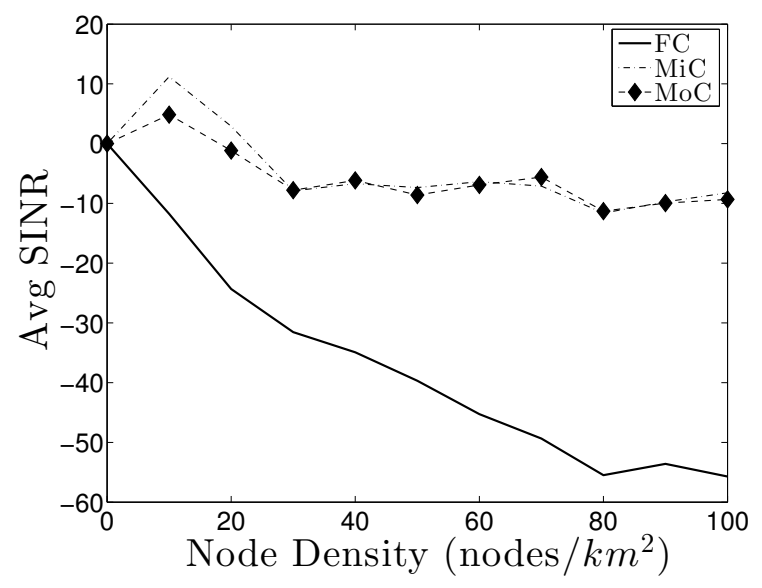

Fig. 5. Average SINR as a function of number of nodes.

value of the throughput increases. The choice of the weighting factors values depends completely on the network requirements and can take any value between 0 and 1. Equation (7) is 


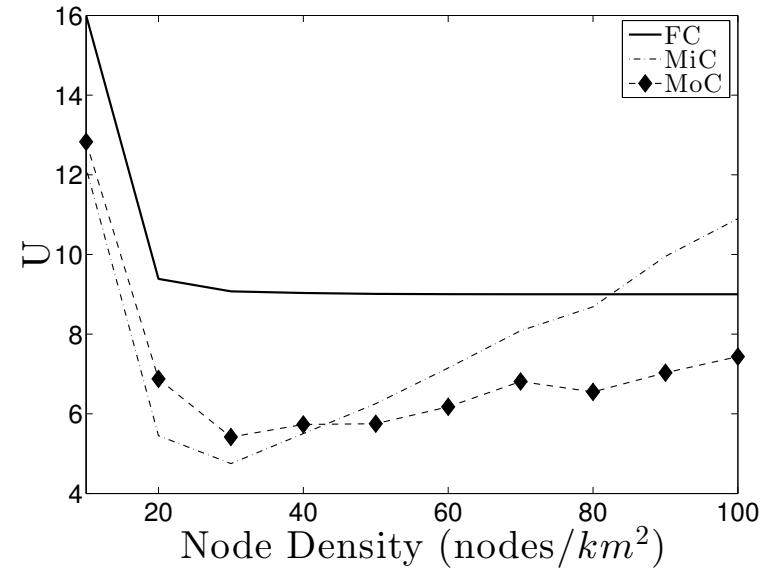

Fig. 6. Utility function as a function of the number of nodes.

suggested to be used in order to have a better understanding of the overall performance tradeoff of a network, but it is necessary to adjust the weighting factors depending on the specific network studied.

Fig. 6 shows the result obtained according to the scenarios analysed in this paper. The best approach, while the nodes density is still under a certain limit, is clearly the FC. Over a particular number of nodes in the network, in this case 40 nodes within a $500 \mathrm{~m}^{2}$ area, it is better to apply power adjustments. The minimally connected topology has the better performance when the network becomes denser. The MoC topology shows to have increasing performance as well. Note that in order to choose a suitable topology, it is fundamental to consider the given constraints of the network; we do so by giving different values to the weight factors.

\section{CONCLUSION}

In this paper, we have studied the problem of topology control of an ad-hoc network through power adjustment. It is well known that network topology in turn determines the connectivity among nodes and the number of hops between any two nodes. The main goal of the work has been to understand the tradeoffs among the transmitting power, the SINR and the number of hops between nodes, where the SINR and the number of hops are highly related to the overall network data rate (throughput) and packet delay. To understand the tradeoffs, we used the fully connected network as a baseline for comparison. Another extreme topology is the minimally connected network, for which we have devised an algorithm to obtain. The third topology obtained by power adjustment provided moderate connectivity among nodes. These three topologies represent a wide range of scenarios, which have been used to portray the possible tradeoffs among transmission power, SINR and hop count. Our results reveal that adjusting just the transmission power is a very effective method to tradeoff these performance parameters; to meet the specific needs of the network users.

Future extensions of this work will be to use more constraints on the connections establishment and also to include a study on high mobile nodes. This work serves as a first step in studying communication connectivity of VANETs.

\section{ACKNOWLEDGMENT}

This work is funded by Defence Science \& Technology Laboratory (DSTL), National UK PhD programme.

\section{REFERENCES}

[1] R. Ramanathan and R. Rosales-Hain, "Topology control of multihop wireless networks using transmit power adjustment," in Nineteenth Annual Joint Conference of the IEEE Computer and Communications Societies (INFOCOM 2000), vol. 2, 2000, pp. 404-413.

[2] L. Hu, "Topology control for multihop packet radio networks," IEEE Transactions on Communications, vol. 41, no. 10, pp. 1474-1481, 1993.

[3] T. A. ElBatt, S. V. Krishnamurthy, D. Connors, and S. Dao, "Power management for throughput enhancement in wireless ad-hoc networks," in IEEE International Conference on Communications (ICC 2000), vol. 3, 2000, pp. 1506-1513.

[4] V. Rodoplu and T. H. Meng, "Minimum energy mobile wireless networks," IEEE Journal on Selected Areas in Communications, vol. 17 no. 8, pp. 1333-1344, 1999.

[5] A. Ephremides, V. Angelakis, and A. Traganitis, "SINR-Based Ad-Hoc Networking," in IEEE 17th International Symposium on Personal, Indoor and Mobile Radio Communications, 2006, pp. 1-5.

[6] G. Quansheng, J. Shengming, D. Quan-Long, and W. Gang, "Impact of topology control on capacity of wireless ad hoc networks," in 11th IEEE Singapore International Conference on Communication Systems (ICCS 2008), 2008, pp. 588-592.

[7] G. Yan, J. C. Hou, and N. Hoang, "Topology control for maintaining network connectivity and maximizing network capacity under the physical model," in The 27th Conference on Computer Communications (INFOCOM 2008), 2008, pp. 1013-1021. 